\title{
Numerical investigation of shock wave attenuation in channels using water obstacles
}

\author{
Q. Wan · R. Deiterding · V. Eliasson
}

Received: date / Accepted: date

\begin{abstract}
Here, short duration direct numerical simulations of shock water cylinder interaction in a two-dimensional channel are conducted to study shock wave attenuation at time scales smaller than the cylinder convection time. Four different cylinder configurations, i.e., $1 \times 1,2 \times 2,3 \times 3$, and $4 \times 4$, are considered, where the total volume of water is kept constant throughout all the cases. Meanwhile, the incident shock Mach number was varied from 1.1-1.4. The physical motion of the water cylinders is quantitatively studied. Results show that the center-of-mass velocity increases faster for the cases with more cylinders. In the early stage of breakup, the transfer rate of kinetic energy from the shock-induced flow to the water cylinders increases as the number
\end{abstract}

This study was supported by the National Science Foundation (NSF) under grant No. CBET-1437412.

Q. Wan

Department of Aerospace

and Mechanical Engineering

University of Southern California

Los Angeles, CA 90089-1189

Present address:

Institute of Manned Space System Engineering

China Academy of Space Technology

Beijing, 100094, China

R. Deiterding

Aerodynamics and Flight

Mechanics Research Group

University of Southampton

Southampton SO17 1BJ, UK

V. Eliasson

Department of Structural Engineering

University of California, San Diego,

La Jolla, CA 92093-0085, USA

E-mail: eliasson@ucsd.edu

Phone: +1 858 534-5928 
of cylinders increases. Further, comparing the cases of different incident shock Mach numbers, higher center-of-mass velocity is induced for the cases of lower incident shock Mach numbers. Moreover, the pressure and impulse changes upstream and downstream of the cylinder matrices are tracked as a quantitative evaluation of the shock attenuation.

Keywords shock-water interaction · multi-phase flow · shock attenuation · impulse

\section{Introduction}

Research on shock wave mitigation is closely related to the development of effective protection and disaster control, and it is motivated by the catastrophic damage that may be caused by shock waves. Overall, the approaches to attenuate a shock wave can be divided into three categories, in terms of mechanism, which are attenuating the shock wave by (1) breaking the incident shock wave into multiple shocks with different arrival times, (2) dissipating energy through viscosity, and (3) transferring kinetic energy of the shock-induced flow to the potential energy of a solid or liquid barrier. Attenuating a shock wave using rigid obstacles in a two-dimensional channel has been numerically studied by Chaudhuri et al. (2013). Obstacles of different shapes, i.e., cylindrical, square and triangular, were placed in either staggered or non-staggered matrix forms. The pressure evolution upstream and downstream of the matrices was monitored as an evaluation for the attenuation effect. Results showed that the staggered matrix of reversed triangular prisms most efficiently attenuated the incident shock wave. More recently, Wan and Eliasson (2015) numerically investigated the shock attenuation ability of a shock focusing geometry. Square and cylindrical rigid obstacles were equidistantly arranged along the edge of a logarithmic spiral, and fixed into a channel. Though arrival of both reflected and transmitted shock waves was effectively delayed, the peak pressure behind the reflected shock remained high.

Water has the potential to be successfully used to attenuate shock waves for several reasons. Water is relatively easy to obtain and environmentally friendly. Water has a large heat capacity, where a large amount of heat could be absorbed by water when mitigating the blast wave. During a TNT explosion, typical heat released is $980 \mathrm{cal} / \mathrm{g}$, while 539 calories are required to vaporize 1 gram of water (Shin et al., 1998). On the other hand, taking account of the environment inside an underground mining structure, solid barriers are hard to install in the narrow channels and may block the lifesaving path during an explosion if collapsed. In comparison, a large bulk of water will be broken into small droplets when impacted by a shock wave, but it will not block the channel due to its fluidic properties. Water can be used in different forms, such as mists, sprays and bulk, to mitigate shock and blast waves (Kailasanath et al., 2002). Part of the kinetic energy from shock-induced flow will transfer to the water by breaking up larger droplets into finer mist, therefore the intensity of secondary shocks could be reduced. Furthermore, due to the existence of 
water, possibly occurring chemical reactions behind the shock front are delayed and slowed down, as water could cover the surface of the flammable material and cut off its contact with the air.

The Weber number is a dimensionless number used to describe the relative importance of the fluid inertia compared to its surface tension. Breakup of a Newtonian droplet has been divided into five different regimes in terms of Weber number: vibrational, bag, bag-and-stamen (or multi-mode), shear or sheet stripping, and catastrophic (Pilch and Erdman, 1987; Guildenbecher et al., 2009). The catastrophic breakup regime has been further divided into wave-crest stripping and catastrophic by some researchers (Pilch and Erdman, 1987; Nomura et al., 2001). Typically, vibrational, bag, and bag-and-stamen types of breakup occur for We $<40$ (Gelfand, 1996), though the vibrational type is not always observed. A shear sheet stripping mode has been observed for $40<\mathrm{We}<10^{3}$. In this case a thin film is continuously stripped off from the droplet surface and quickly collapses after being removed. The catastrophic mode has been found for $10^{3}<\mathrm{We}<10^{5}$, where the droplet surface is crinkled by the flow and forms a small number of large fragments that in turn break up into smaller droplets.

Previous studies of liquid droplet breakup by shock wave involve both numerical and experimental work (Theofanous, 2011). Ranger and Nicholls (1969) experimentally studied shattering of water droplets under shock waves with Mach number $M_{s}=1.3-3.5$ in air. Results showed that the breakup of droplets was mostly caused by the induced flow behind the incident shock wave. In addition, the breakup time was found to be proportional to the deforming droplet diameter, and inversely proportional to the droplet velocity. Wierzba and Takayama (1988) were among the first to use an experimental method to precisely study the breakup mechanism of liquid droplets under shock impact. Results revealed that the stripping-type breakup of water droplets can be divided into four steps, i.e., (1) liquid surface disruption, (2) droplet deformation, (3) continuous stripping of microdrops, and (4) breakup of the remaining part into relative large fragments as well as secondary stripping of microdrops from the fragments. Later, Yoshida and Takayama (1990) further investigated interactions between shock wave and liquid droplet experimentally. Results showed that during the impact of shock waves, the water droplet was first stretched in its horizontal direction, and then mist formed due to the separation of the resulting boundary layer.

The physical motion of a single water droplet breaking up by a shock wave has been numerically studied in recent years. Meng and Colonius (2015) simulated the early stage of the breakup process of a single water cylinder by a planar shock wave. The post-shock gas velocity was varied from subsonic to supersonic, and the wake region for the supersonic case was narrower than the subsonic case, yet no significant difference was found regarding the flow features. Though the simulations were conducted without viscosity and liquid surface tension, numerical schlieren images for early breakup stage were comparable with previous experimental work of Igra and Takayama (2001a). Quantitative features, such as center-of-mass drift, velocity and centerline 
width, showed good agreement compared to the experimental results from Igra and Takayama (2001b) and numerical results from Chen (2008). Also, results showed that stronger incident shock waves induced higher center-ofmass velocity of the water cylinder. Sembian et al. (2016) studied the wave propagation inside a water cylinder during the impact of a shock wave with Mach numbers 1.75 and 2.4. Cavitation bubble clouds forming inside the water cylinder were captured using the shadowgraph technique, but were only observed in the Mach 2.4 case, which was possibly due to the negative gauge pressure that was recorded inside the water cylinder.

Propagation of a planar shock wave through a cloud of small water droplets has been experimentally studied in the recent decade, yet the numerical modeling for such problem is hard to conduct due to the large computational cost of the simulations. Jourdan et al. (2010) experimentally investigated the mitigation of shock wave passing through a cloud of water droplets. Experiments were conducted using a vertical shock tube, and water droplets were carefully controlled to be 120,250 and $500 \mu \mathrm{m}$ in diameter. The attenuation of the shock was characterized by reducing the peak pressure after passing through the water cloud. Results showed that the attenuation is negligible for droplets of $120 \mu \mathrm{m}$, while droplets of $500 \mu \mathrm{m}$ significantly reduced the peak pressure behind the transmitted shock by $65 \%$. Chauvin et al. (2011) conducted similar experiments using different heights of water droplets clouds while the droplet diameter was fixed. Further, it has been shown that the major factor of shock wave mitigation is the exchange surface area of droplet atomization, which is defined as the effective area of the droplets crossed by the shock wave at a given location, non-dimensionalized by the cross section of the shock tube. In general, when a planar shock wave propagates through a cloud of water droplets, a transmitted shock forms downstream of the droplet cloud. Simultaneously, a reflected shock traverses in the opposite direction upstream of the droplets, as shown in Fig. 1(a). The corresponding pressure change at the downstream pressure probe is depicted in Fig. 1(b).

Cylindrical water obstacles have also been studied experimentally by Igra and Takayama (2003). Water cylinders were placed in a tandem configuration and results were presented in terms of acceleration terms and drag coefficients. The tandem configuration was also compared to that of a single water cylinder, and results showed that the single water cylinder behaved "virtually the same" as the front cylinder in the tandem case. The rear cylinder experienced less displacement, less acceleration and had a lower drag coefficient compared to the front cylinder.

The aim of this work is to quantitatively study the attenuation of a shock wave in a two-dimensional channel filled with air at initially atmospheric conditions containing water cylinders during a very early stage as the shock wave passes through the region with water cylinders. The water cylinders are placed in different matrix forms, while the total amount of water is kept constant. The numerical model is a multi-scale model leading to an accurate volumebased homogenization of the two phases in the moment that interfaces cannot be resolved on the mesh. The goal is to understand the interactions between 
shock waves and water cylinders, and precisely calculate the energy exchange between the two-phase flow. In addition, by comparing the pressure change upstream and downstream, one can quantitatively compare the attenuation effects of different water cylinder configurations. The current study is organized in the following way: The numerical method and constraints are described in section 2. Then, results and analysis are presented in section 3. In particular, the physical motion and flow features are presented in section 3.1, and the transfer of kinetic energy from air to water is discussed in section 3.2. The behavior of cylinder matrices under different incident shock Mach numbers is shown in section 3.3. The shock attenuation is analysed in section 3.5, and comparison between the water cylinder matrices and solid obstacle cases is further discussed in section 3.6. At last, conclusions are drawn in section 4 .

\section{Numerical approach}

\subsection{Simulation setup}

In a two-dimensional channel, propagation of a planar incident shock wave coming from left to right through water cylinders placed in matrix forms is considered, as depicted in Fig. 2. Four different cylinders placements, i.e., $1 \times 1$, $2 \times 2,3 \times 3$, and $4 \times 4$ cylinders, are used. The total amount of water, which is indicated by the total cross section area of the water cylinders, is kept constant in all the cases. The diameter of the cylinder in the $1 \times 1$ case, shown in Fig. 2(a), is chosen to be $d_{0}=6 \mathrm{~mm}$, and the cylinder diameters for the remaining cases are determined to maintain a constant total amount of water. In particular, water cylinders are strictly placed within the $w \times w$ square region, where the center-of-mass is initially located at the origin. Top and bottom boundaries are parallel and represent channel walls, where reflecting characteristic boundary conditions are applied. The left side is the inflow; the right side is the outflow. Atmospheric conditions are applied to the water cylinders and the air ahead of the incident shock wave, i.e., $\rho_{g}^{a}=1.204 \mathrm{~kg} / \mathrm{m}^{3}$, $\rho_{l}=1000 \mathrm{~kg} / \mathrm{m}^{3}, p_{g}^{a}=p_{l}=101325 \mathrm{~Pa}$. The effect of gravity is negligible and not taken into account in the current study. As shown in Fig. 2(a), $S_{1}$ and $S_{2}$ are the locations where the pressure values are recorded, which will be referred as pressure probe 1 and pressure probe 2, respectively. Both pressure probes are placed at the same locations for all the configurations. The simulation time is non-dimensionalized using equation (1), where $\tau_{d}$ denotes the characteristic deformation time (Gelfand, 1996; Chauvin et al., 2011) of the cylinder associated with its diameter, as shown in Eqn. (1),

$$
t^{*}=\frac{t}{\tau_{d}}, \tau_{d}=\frac{\phi_{d}}{u_{g}^{s}} \sqrt{\frac{\rho_{l}}{\rho_{g}^{s}}},
$$

where $t$ is the actual time, $\phi_{d}$ represents the water cylinder diameter in general, $\phi_{d}=d_{0}$ in the $1 \times 1$ case, $u_{g}^{s}$ and $\rho_{g}^{s}$ denote shock-induced flow velocity and density, respectively. 
Table 1 Summary of incident shock-induced flow conditions for simulated Mach numbers

\begin{tabular}{cccc}
\hline$M_{s}$ & $p_{g}^{s}[\mathrm{kPa}]$ & $\rho_{g}^{s}\left[\mathrm{~kg} / \mathrm{m}^{3}\right]$ & $u_{g}^{s}[\mathrm{~m} / \mathrm{s}]$ \\
\hline 1.1 & 126.2 & 1.408 & 54.61 \\
1.2 & 153.3 & 1.615 & 104.9 \\
1.3 & 182.9 & 1.825 & 151.8 \\
1.4 & 214.8 & 2.034 & 196.1 \\
\hline
\end{tabular}

Incident shock Mach numbers are varied from $M_{s}=1.1$ to $M_{s}=1.4$ with increment 0.1 . For each specific incident shock Mach number, the corresponding shock-induced flow pressure, $p_{g}^{s}$, density, $\rho_{g}^{s}$, and velocity, $u_{g}^{s}$, are shown in Tab. 1.

\subsection{Governing equations}

The set of used governing equations are based on a multi-component model that involves the phase volume fractions $\alpha_{i}$ with $\sum_{i=1}^{m} \alpha_{i}=1$. Mixture quantities are described as

$$
\rho=\sum_{i=1}^{m} \alpha_{i} \rho_{i}, \quad \rho \mathbf{u}=\sum_{i=1}^{m} \alpha_{i} \rho_{i} \mathbf{u}_{i}, \quad \rho e=\sum_{i=1}^{m} \alpha_{i} \rho_{i} e_{i} .
$$

Multiple fluids are represented as mostly separated phases that can be described by a single set of two-dimensional inviscid Euler equations

$$
\begin{gathered}
\partial_{t} \rho+\nabla \cdot(\rho \mathbf{u})=0, \\
\partial_{t}(\rho \mathbf{u})+\nabla \cdot(\rho \mathbf{u} \otimes \mathbf{u})+\nabla p=0, \\
\partial_{t}(\rho E)+\nabla \cdot((\rho E+p) \mathbf{u})=0,
\end{gathered}
$$

which represent the conservation of mixture mass, mixture momentum and total energy without any body forces. Here, $\mathbf{u}$ is the velocity vector and $E:=$ $e+\frac{1}{2} \mathbf{u}^{T} \mathbf{u}$ the specific total energy. Applying a stiffened gas equation of state

$$
p_{i}=\left(\gamma_{i}-1\right) \rho_{i} e_{i}-\gamma_{i} p_{i, \infty}
$$

the total hydrodynamic pressure $p$ and $p_{\infty}$ of the mixture are given as

$$
\frac{p}{\gamma-1}=\sum_{i=1}^{m} \frac{\alpha_{i} p_{i}}{\gamma_{i}-1}, \quad \frac{\gamma p_{\infty}}{\gamma-1}=\sum_{i=1}^{m} \frac{\alpha_{i} \gamma_{i} p_{i, \infty}}{\gamma_{i}-1} .
$$

Assuming a constant specific heat at constant volume, $c_{v, i}$, the internal energy in each fluid can be related to the partial temperature $T_{i}$ as Flåtten et al. (2011)

$$
e_{i}=c_{v, i} T_{i}+\frac{p_{i, \infty}}{\rho_{i}}
$$


which allows expressing Eqn. (6) as

$$
p_{i}=\left(\gamma_{i}-1\right) \rho_{i} c_{v, i} T_{i}-p_{i, \infty} .
$$

Instead of using propagation equations of $\alpha_{i}$ to distinguish the components, the approach of Shyue (1998) has been adopted to supplement equations (3)(5) with the two advection equations

$$
\begin{aligned}
& \frac{\partial}{\partial t}\left(\frac{1}{\gamma-1}\right)+\mathbf{u} \cdot \nabla\left(\frac{1}{\gamma-1}\right)=0, \\
& \frac{\partial}{\partial t}\left(\frac{\gamma p_{\infty}}{\gamma-1}\right)+\mathbf{u} \cdot \nabla\left(\frac{\gamma p_{\infty}}{\gamma-1}\right)=0 .
\end{aligned}
$$

Although this description is applicable only to two-component systems, i.e., $m=2$, the benefit is that the direct utilization of equations (10) and (11) in the governing equations and therefore direct discretization together with (3)(5) is the simplest remedy (Shyue, 2006) to the otherwise intrinsic problem of nonphysical numerical pressure oscillations at interfaces with vastly different parameters $\gamma_{i}$ and $p_{i, \infty}$ (Abgrall and Karni, 2001). Here, the parameters for water were defined as $\gamma_{l}=6.12$ and $p_{l, \infty}=343.44 \mathrm{MPa}$ (Meng and Colonius, 2015 ), resulting in an ambient speed of sound of $a_{0}=1450 \mathrm{~m} / \mathrm{s}$ and $\gamma_{g}=1.4$ and $p_{g, \infty}=0$ for air.

Note that the described model considers phases as mixed within a cell and thereby represents bubbles or droplets below the grid scale by an alteration of the volume fractions $\alpha_{i}$. Based on the relation

$$
\frac{1}{\gamma-1}=\sum_{i=1}^{m} \frac{\alpha_{i}}{\gamma_{i}-1}
$$

that is imposed in addition, cf. (Shyue, 1998, 2006), it is straightforward to evaluate of $\alpha_{i}$ for the case $m=2$ from the mixture quantity $1 /(\gamma-1)$ when solving for equations (3)-(5) plus (10) and (11).

\subsection{Numerical method}

A time-explicit finite volume approach is used to discretize the governing equations with the second-order accurate wave propagation method (LeVeque, 2002). This method is designed for schemes in flux-difference splitting form and can handle the conservative equations (3)-(5) with an approximate Riemann solver as well as the non-conservative advection equations (10) and (11). In order to cope with realistic density differences across the air-water interface, the Harten-Lax-van Leer with contact (HLLC) scheme by Toro et al. (1994) is used as approximate Riemann solver. An extended version of the HLLC scheme, tailored for the above set of governing equations for two-phase flow and smoothly incorporated into the Wave Propagation approach had been specially derived (Deiterding et al., 2009). The robustness of the HLLC approach allows using the accurate densities for water and air. 
In order to enable technically relevant computations, the sketched finite volume method has been incorporated into the Cartesian adaptive mesh refinement solver system AMROC V2.0 (Deiterding, 2011) within the freely available fluid-structure coupling software Virtual Test Facility (Deiterding, 2005). The consideration of geometrically complex boundaries in AMROC is achieved by a generic and discretization-independent level set technique. Based on signed distance information stored in the level set function, reflective wall boundary conditions are constructed by inter- and extrapolation operations in cells adjacent to the boundary, but deemed outside of the fluid domain, before the Cartesian discretization is employed to compute the next time step (Deiterding, 2009). Block-based mesh refinement is applied and regenerated consecutively at run-time to mitigate inaccuracies from the boundary approximation as well as to capture essential flow features, like shock waves and material interfaces with increased resolution (Deiterding, 2011). In the current study, three adaptive mesh refinement (AMR) levels are chosen and a refinement factor of 2 is used. A coarse background grid of resolution $1220 \times 124$ is created so after mesh refinement, the effective grid resolution is $4880 \times 496$. Time step refinement by the same factor as the spatial refinement ensures that the stability condition of the explicit finite volume scheme is in principle satisfied on all refinement levels.

Validation simulations for the sketched dynamically adaptive two-phase HLLC method are provided by Perotti et al. (2013), where elastic deformations of tubes from shock waves in water have been analyzed meticulously. Further verification and validation simulations for water-hammer and underwater explosion driven plastic deformation and rupture of metallic plates with twophase flow and empirical cavitation modeling are given by Cirak et al. (2007) and Deiterding et al. (2009). Lastly, for further validation and verification, a combined numerical and experimental study on different shock wave reflection patterns, transition angles and triple point trajectory angles for oblique shock wave reflection off a water wedge at different inclination angles and for a range of shock Mach numbers is provided by Wan et al. (2017).

\subsection{Numerical comparison}

Before starting the simulations of multiple cylinder matrices, a comparison of the numerical approach is conducted by reproducing the results from Igra and Takayama (2001b) and Meng and Colonius (2015), which showed the breakup process of a single water cylinder under high-speed flow. The simulation setup is described in Meng and Colonius (2015), as shown in Fig. 3, where a planar shock wave with Mach number $M_{s}=1.47$ is traveling from left to right. Symmetric boundary conditions are applied to the bottom boundary. Left side is inflow, and right and top sides are outflow.

The water cylinder's leading edge drift, i.e., forward stagnation point, and cylinder's centerline width are traced and shown in figures 4(a) and (b). A threshold of the liquid volume fraction, $\alpha_{T}$, was used to bound the water cylin- 
der, and all the cells with a liquid volume fraction $\alpha_{l} \geq \alpha_{T}$ are counted as part of the water cylinder. The displacement of the leading edge, $\Delta x$, and centerline width, $w_{c}$, are non-dimensionalized by the cylinder diameter, $d_{e}=4.8 \mathrm{~mm}$, and the timeline is non-dimensionalized by the characteristic deformation time regarding $d_{e}$ using equation (1). By comparison, our results agree well with both the numerical results from Meng and Colonius (2015) and experimental measurements from Igra and Takayama (2001b).

In addition to the numerical comparison, a convergence study is conducted to further validate the mesh resolution. The $1 \times 1$ water cylinder case is simulated using five different background grid resolutions, i.e., $152 \times 16,304 \times 30$, $610 \times 62,1220 \times 124$ and $2440 \times 248$, with respectively three AMR levels refined by a factor of 2 each. Fig. 5 shows the center-of-mass drift obtained using different grid sizes. Convergent results are achieved as the grid resolution is refined and hence the background coarse mesh $1220 \times 124$ is applied in the rest of the simulations throughout this paper, cf. Section 2.3 .

\section{Results and analysis of water cylinder matrices breakup}

The physical motion of water cylinders, i.e., center-of-mass drift, center-ofmass velocity, and kinetic energy exchange between air and water, under shock wave impact is quantitatively studied. Further, the attenuation of shock wave is characterized by the pressure change in the flow field.

\subsection{Water cylinders motion and flow features}

Before looking into the shock attenuation by water cylinders, a physical understanding of the behavior and motion of the water cylinder matrix under the impact of a planar incident shock wave is informative. Here, a qualitative comparison for the flow features is conducted. Initially, the water cylinders are located as shown in Fig. 2. The density contours at four sequential time instants are visualized to show the propagation of a $M_{s}=1.3$ shock wave through the water cylinder matrix, as depicted in figures 6-7. The threshold of the visualized density is chosen to be in the range $1.2-16 \mathrm{~kg} / \mathrm{m}^{3}$ so that more details of the flow features can be captured. Also, the density contour is a good representation of the mass flux of the flow field. As seen from the density contour plots, there is no symmetry in the upper and lower half planes. This is caused by initial conditions not being $100 \%$ symmetric, and quite common because all high-resolution solvers eventually show symmetry breaking at unstable shear layers (only very large numerical diffusion could stabilize the physically unstable process).

As reviewed earlier, the incident shock wave itself does little effect to the water droplet deformation (Ranger and Nicholls, 1969). In Fig. 6(a), the incident shock waves have already propagated through the cylinder matrices, and are outside the frame. For the $1 \times 1$ cylinder case, the cylindrical shape is 
not much affected and can be recognized. For the $2 \times 2$ water cylinders case, the deformation of the first and second columns is slightly different. In the left column, the water cylinder on top is stretched in horizontal direction, simultaneously a clockwise vortex is formed at the upper side of the back of the water cylinder, as shown in Fig. 8, which has been previously reported by Meng and Colonius (2015). Similarly, a counter-clockwise vortex, shown in Fig. 8, is formed at the lower side. These vorticity streams induce shear stress that deforms the water cylinder of the right column, which results in a pointed shape at the front side of the water cylinder. Similar deformation modes can be found in the $3 \times 3$ and $4 \times 4$ cylinders cases.

In figures $6(\mathrm{~b})$ and $7(\mathrm{a})$, the cylinder of the $1 \times 1$ case is further stretched in width. Especially in Fig. 7(a), a liquid film is formed that gets peeled away from the cylinder surface, which is a typical phenomenon in the shear stripping deformation mode. For the $2 \times 2,3 \times 3$ and $4 \times 4$ water cylinders cases, the water cylinders of different columns start to merge. Water cylinders at the right columns are first stretched in the horizontal direction, where the front side is moving toward the back side of the left column.

The cylinders of smaller sizes are more deformed, which is expected because the characteristic deformation time of a cylinder, shown in Eqn. (1), is proportional to the cylinder diameter. As shown in Fig. 7(b), the main body of the water cylinder for the $1 \times 1$ case is maintained at a similar size as the original, while for the other three cases, cylinders are stretching and merging, and vortex shedding in the wake region occurs, which makes the flow field highly turbulent and the cylindrical shapes are not recognizable anymore.

Comparing the density contours of the four different cylinder configurations in figures 6-7, it can be observed that the mass transportation mode is different as the number of cylinders increases. For the $1 \times 1$ configuration, most of the water cylinder mass is maintained near its original circular shape, while for the $4 \times 4$ configuration, water cylinders are spread all over the wake region. Thus, besides the qualitative visualization of the density contour, the physical motion of water cylinders is quantitatively studied.

Since the total volume of water is kept constant in all the cases, and the location of the center-of-mass for each case is initiated at the same origin, this makes it possible to investigate the difference of cylinder movement under directly comparable conditions. The displacement and velocity of the centerof-mass of water cylinder matrices are evaluated using the method described in Meng and Colonius (2015) by evaluating

$$
x_{c}=\frac{\int \alpha_{l} \rho_{l} x d V}{\int \alpha_{l} \rho_{l} d V}, u_{c}=\frac{\int \alpha_{l} \rho_{l} u d V}{\int \alpha_{l} \rho_{l} d V},
$$

where the volume is integrated over the entire computational domain. The calculations naturally minimize noise produced by the finite difference approximation, but still assume zero mass flux across the computational boundaries (Meng and Colonius, 2015). Therefore, once liquid mass crosses through the computational boundaries, equation (13) is no longer valid. 
Following the procedure in Meng and Colonius (2015), the center-of-mass drift (also called water cylinder matrix streamwise displacement) of the four cylinder configurations, $1 \times 1,2 \times 2,3 \times 3$ and $4 \times 4$, under incident shock Mach number $M_{s}=1.3$ is evaluated and shown in Fig. 9. The time is non-dimensionalized using equation (1), where the water cylinder diameter is chosen as the diameter of the $1 \times 1$ case, $\phi_{d}=d_{0}$. Displacement of the center-of-mass, $x_{c}$, is also non-dimensionalized using $d_{0}$. As can be seen from Fig. 9, for each individual case, the displacement increases monotonically over time. Comparing cases of different cylinder configurations, the displacement increases as the number of water cylinders increases.

The corresponding center-of-mass velocity is calculated using Eqn. (13), which is not merely taking the derivatives of the center-of-mass drift. The resulting center-of-mass velocity is shown in Fig. 10. Generally, velocity is monotonically increasing over time for each case. Particularly, at the early stage, the velocity profile for each case grows linearly. When water cylinders have not deformed significantly, the pushing force from the induced flow is almost constant, which leads to a relatively stable and unaltered acceleration of the water cylinder matrix. After a certain time, when liquid filaments start to be stripped from the cylinder edge and move away from its main body, and cylinders of different columns begin to merge together, part of the kinetic energy is lost, which causes instability of the velocity profile. More details regarding the energy exchange between shock-induced flow and water cylinders are discussed in the next section. Additionally, a higher center-of-mass velocity is induced for configurations of more water cylinders.

The above increasing trend of the center-of-mass drift and velocity is expected, because the case with more cylinders has larger exchange surface area, which is defined as the total cylinders' cross section area, i.e.,

$$
S_{e}=n \phi_{d} .
$$

A similar definition is given by Chauvin et al. (2011). In Eqn. (14), $n$ denotes the total number of cylinders. Since the simulations are two-dimensional, the initial direct contact surface area between air and water is the total perimeter of all cylinders, while the exchange surface area can be defined as the total streamwise cross section area, which is the sum of all cylinder diameters. Water cylinders in the cases of larger $S_{e}$ expose more surface area to the air in the stream-wise direction, therefore the cylinders are pushed by larger force under the same initial conditions. The exchange surface areas for the $1 \times 1,2 \times 2,3 \times 3$ and $4 \times 4$ cylinder matrix configurations are $d_{0}, 2 d_{0}, 3 d_{0}$ and $4 d_{0}$, respectively.

\subsection{Energy exchange between air and water}

The total kinetic energy of water cylinder matrices is computed as

$$
E_{k}=\frac{1}{2} \int \alpha_{l} \rho_{l}\left(u^{2}+v^{2}\right) d V
$$


where $u$ and $v$ denote the velocity in each cell, and the volume is integrated over the entire domain. The total kinetic energy of water cylinders, $E_{k}$, is purely transferred from the shock-induced flow. Then, normalizing $E_{k}$ by the total kinetic energy input of the shock-induced flow provides a measure of energy exchange between air and water. The normalization is shown in equation (16). The resulting non-dimensional quantity $E_{n}$ shows the fraction of kinetic energy transferred from air to water, which can also be seen as the energy exchange efficiency,

$$
E_{n}=\frac{E_{k}}{E_{0}}, E_{0}=\frac{1}{2} \rho_{g} u_{g}^{3} H\left(t_{f}-t_{s}\right),
$$

where $E_{0}$ denotes the total input kinetic energy from shock-induced flow, $H$ is the height of the computational domain, here $H=2 d_{0}, t_{f}$ is the final time of the simulation, and $t_{s}$ is the moment that the incident shock wave arrives at the leading edge of the water cylinder matrices.

The trace of the total kinetic energy of water cylinder matrices under incident shock Mach number $M_{s}=1.3$ is shown in Fig. 11. The four time instants shown in Figs. 6-7 are marked here so that connections can be made between the density contours in Fig. 6-7 and the corresponding energy exchange. The timeline of the four cases, denoted as $t_{0}^{*}$, is scaled using equation (1) with the same $\phi_{d}=d_{0}$. In Fig. 11, the slope of the curves denote the kinetic energy transfer rate from air to water. At the early stage, $t_{0}^{*}<0.15$, kinetic energy is transferred faster from air to water for the cases with more water cylinders, because of larger exchange surface area. More energy is taken from the shockinduced flow to simultaneously break up multiple smaller water cylinders than a single larger cylinder. After $146 \mu$ s, water cylinders in the $3 \times 3$ and $4 \times 4$ cases break up quickly, which can be seen in Fig. 6(b), thus the energy curves of the two cases almost collapse in Fig. 11 for $t_{0}^{*}>0.2$. Meanwhile, at $290 \mu \mathrm{s}$, the cylinders in the $2 \times 2$ case mostly maintain their original shape, shown in Fig. 6(b), where the two columns are moving toward each other but have not yet merged. Therefore, the energy exchange for the $2 \times 2$ case is relatively smooth before $290 \mu \mathrm{s}$. After $290 \mu \mathrm{s}$, cylinders in the $2 \times 2$ case merge gradually and cause energy loss.

To summarize, during the simulated time, up to $23 \%$ of kinetic energy input from the shock-induced flow is converted into kinetic energy of water cylinders for the $3 \times 3$ configuration, which is the highest among the four cases. For the other three cases, the input kinetic energy transfer rate is $19 \%, 17 \%$, and $19 \%$ for $1 \times 1,2 \times 2$, and $4 \times 4$ cases, respectively.

\subsection{Effect of incident shock Mach number}

Four different incident shock Mach numbers, $M_{s}=1.1-1.4$ with increments 0.1 , were explored to understand the variation of the cylinders' physical behavior under different induced flow velocities.

The center-of-mass drift in the direction of the incident shock wave for $1 \times 1$ and $3 \times 3$ water cylinder matrices is shown in Fig. 12. The streamwise 
displacements are normalized by the diameter of the $1 \times 1$ water cylinder. The curves describe that the water cylinder matrices move slower, indicated as the curve slopes, as the incident shock Mach number is increased. On the other hand, it can be found that increasing the number of cylinders in the matrix does not make a significant difference to its physical behavior.

The center-of-mass streamwise velocity for $1 \times 1$ and $3 \times 3$ water cylinder matrices is shown in figures $13(\mathrm{a})$ and (b). The evaluated velocities are normalized by respective shock-induced flow velocities. The velocity profiles show a similar trend as the center-of-mass drift, where weaker incident shock waves induced higher cylinder matrix velocities. The resulting velocity trend is thus opposite to the finding of Meng and Colonius (2015), yet still reasonable. The computational domain in the current simulations are bounded with the rigid reflecting walls at upper and lower sides, while in Meng and Colonius (2015), non-reflecting characteristic boundary conditions were applied to the upper wall, as shown in Fig. 3. The gaps between the cylinder matrix and the rigid walls are comparable to the cylinder diameter, so it does not take long time for the incident shock to get deflected by the water cylinders and reflected back from the top and bottom walls. Therefore, when the rounded reflected shock forms, the second column cylinders gets accelerated by the reverberating shocks that reflect off the top and bottom walls, and the induced flow behind the reverberating shock would push back the water cylinders at the first column in the opposite direction of the incident shock wave. The backward pushing pressure increases as the incident shock Mach number increases, and the velocity of the cylinder matrix decreases. The case of $2 \times 2$ cylinder matrix for $M_{s}=1.3$ is illustrated as an example in Fig. 14, while the backward pushing of the reflected shock can be found in the other configurations. On the other hand, in the early stage of the cylinder breakup, the cylinder is acting like a rigid circular obstacle when impacted by a shock wave, which can be seen in Fig. 14.

The kinetic energy exchange rate between air and water for the $1 \times 1$ and $3 \times 3$ cylinder matrices under the four different incident Mach numbers is calculated and non-dimensionalized using equations (15)-(16). Results are shown in figures 15(a) and (b). The slope of the curve indicates the rate of kinetic energy transfer from air to water. Hence, it is clear that the transfer of energy is faster for lower incident shock Mach numbers. This is consistent with the results of center-of-mass velocity shown in Fig. 13. In addition, the energy transfer rate increases (the slope gets steeper) over time for the $1 \times 1$ case. This is because initially the cylinder does not deform significantly, thus not much energy was absorbed. Later, the cylinder is broken gradually, and exposes more surface area to the air, therefore kinetic energy transfer is increased. For the $3 \times 3$ case, the energy curves are not as smooth as the $1 \times 1$ case, which is because more cylinders are merging and an increase in water cylinder-wall reflection cause energy loss. 
3.4 Temperature change

Figure 16 shows the temperature increment of the flow fields around water cylinders under the Mach number $M_{s}=1.3$. In general, the temperature upstream of the cylinders is higher than downstream. This is expected because the gas is expanding isentropically around the obstacles. Also, due to the large heat capacity of the water, the temperature change in the water is much smaller than in the air. In particular, the temperature increment of the water phase is less than $5 \mathrm{~K}$, which is negligible comparing with the temperature change in the air.

\subsection{Shock attenuation}

As discussed earlier, the presence of water cylinders alters the pressure trace downstream of the incident shock wave, which is illustrated in Fig. 1. Although the characteristic peak overpressure, $\Delta p$, may not be the global maximum pressure, it is one quantity that can be used to characterize the transmitted shock wave. Alternatively, the pressure impulse, the time integral of overpressure,

$$
I=\int \Delta p(t) d t
$$

is another measure used to quantify the shock attenuation effect. Therefore, the attenuation of the shock wave is studied in two aspects: (1) the overpressure ratio $\Delta p / p_{g}^{s}$, and (2) the pressure impulse. Specifically, the overpressure, $\Delta p$, is picked as $p-p_{g}^{s}$ upstream due to the passage of the incident shock wave, and $p-p_{g}^{a}$ downstream.

Upstream of the water cylinders, under the incident shock Mach $M_{s}=1.3$, the traces of overpressure and impulse evaluated at the location $S_{1}$ (see Fig. 2) are shown in Figs. 17(a) and (b), where the overpressure is normalized by the pressure derived from the case without cylinders. In order to avoid that the overpressure evaluated at the center point of $S_{1}$ is very different from the overpressure close to the top and bottom walls, the averaged overpressure along $S_{1}$ is also recorded. The averaged overpressure is shown as the solid line in Fig. 17, and the recorded overpressure from the center point of $S_{1}$ is shown using the dashed line. Comparing with the evaluation at the center point, the calculation of the averaged overpressure removes the noises and ensures that the pressure trend elsewhere is consistent. The spatially averaged overpressure shows a similar trend with the evaluation at the centerline, while unnecessary noise is to some extent reduced in the averaged results. The pressure probe 1 is located behind the incident shock wave at the beginning of the simulation, so the overpressure evaluated at $t_{0}^{*}=0$ is the shock-induced pressure. The overpressure behind the reflected shock is used to calculate the impulse, and the result is shown in Fig. 17(b). The spatially averaged results overlap those evaluated along the centerline. By the end of the simulated time, the pressure impulse of the $4 \times 4$ case is $43.0 \mathrm{~Pa} \cdot \mathrm{s}$, which is the highest among the four 
cases. The evaluated impulses for the $1 \times 1,2 \times 2$ and $3 \times 3$ cases are $76 \%, 87 \%$ and $94 \%$, respectively, compared to the result from the $4 \times 4$ case.

Similarly, the normalized overpressure obtained at the downstream location is shown in Fig. 18(a). The solid lines show the trace of the averaged overpressure along $S_{2}$, and the corresponding dashed lines show the evaluation at the center point of $S_{2}$. For each individual case, a jump in overpressure is followed by an underpressure, caused by the presence of expansion waves. Further, comparing the cases of different configurations, the duration of the first overpressure jump decreases as the number of cylinders increases. Integrating the overpressure leads to the pressure impulse shown in Fig. 18(b). The noise is further eliminated by integration. As the transmitted shock front reaches the pressure probe, the impulse values obtained for each configuration increase when $\Delta p$ is positive. Later, the impulse either increases with a lower rate or it decreases due to the existence of underpressure. Comparing with the theoretical solution of the case without water cylinders, obviously the presence of cylinder matrices significantly reduces the impulse downstream. By the end of the simulated time, the pressure impulses for $1 \times 1,2 \times 2,3 \times 3$ and $4 \times 4$ cases are $27 \%, 26 \%, 27 \%$ and $23 \%$ compared to the case without any cylinders.

\subsection{Comparison with the solid obstacle case}

In order to further characterize the shock attenuation effect due to water cylinders deformation and kinetic energy transfer, the propagation of a $M_{s}=$ 1.3 shock wave through solid obstacle matrices with the same geometrical setup as Fig. 2 is simulated. Specifically, the solid obstacles are of the same size as water cylinders, and placed at the same corresponding locations, but neither deformable nor movable in the simulations. Overpressure and impulse are traced at the same probe location mentioned as in section 3.5, while only the spatial averaged evaluation along $S_{1}$ and $S_{2}$ are shown.

Since deformation is not considered for the solid obstacle cases, dimensional time is used. At the upstream location, a comparison of the overpressure traces for the $1 \times 1$ and $4 \times 4$ cases is shown in Fig. 19(a). For the $1 \times 1$ configuration, the overpressure traces of the solid obstacle and water cylinder cases overlap at time $t<0.14 \mathrm{~ms}$, when the cylinder has not yet deformed. Later, the overpressure of the water cylinder case gradually rises above the solid obstacle case as the cylinder is more stretched in its horizontal direction. A similar trend for the overpressure traces can be found for the $4 \times 4$ case, yet the duration of overlapped overpressure is shorter than in the $1 \times 1$ case. The corresponding impulse regarding the overpressure is shown in Fig. 19(b). By the end of the simulated time, for the $1 \times 1$ configuration, the impulse of the solid obstacle case is $45 \%$ of the water cylinder case. For the $4 \times 4$ configuration, the impulse of the solid obstacle case is $50 \%$ of the water cylinders case.

Downstream of the water cylinder matrices, the normalized overpressure is shown in Fig. 20(a). An enhanced attenuation effect is achieved for the water cylinder cases, whose overpressures drop faster. The attenuation effect 
is also reflected in the corresponding impulse traces, as shown in Fig. 20(b). By the end of the simulated time, the impulse of the water cylinder cases is $40 \%$ and $31 \%$ of the solid obstacle cases respectively for the $1 \times 1$ and the $4 \times 4$ configurations.

\section{Conclusions}

The propagation of a planar incident shock wave through a two-dimensional channel, which is blocked by water cylinder matrices of four different configurations, i.e., $1 \times 1,2 \times 2,3 \times 3$, and $4 \times 4$, has been modeled numerically. The total volume of water has been kept constant, and the locations of the center-ofmass were initiated at the same point throughout all the configurations. First, the physical motion of water cylinder matrices and the flow features have been investigated. Quantitative evaluation of the center-of-mass drift and velocity were conducted using the method previously reported by Meng and Colonius (2015). The first set of simulations were performed with incident shock Mach number $M_{s}=1.3$. Results show that the center-of-mass drift increases monotonically for each case. Specifically, velocity increases faster for the cases with more cylinders. Further, the transfer of kinetic energy from the shock-induced flow to the water cylinders has been quantified. To the best of the authors' knowledge, this is the first work that quantitatively calculates the energy exchange between shock waves and water cylinders. Our results show that the transfer rate of kinetic energy, in the early stage, from the shock-induced flow to the water cylinders, increases as the cylinders' number is increased. However, as the cylinders deform further, and different cylinder columns merge with one another, the energy trace becomes unpredictable and eventually collapses. By the end of the simulated time, up to $23 \%$ of the kinetic energy input from the shock-induced flow has been converted to kinetic energy of the water cylinders in the $3 \times 3$ case, which is the highest transfer rate among the four considered cases.

Further, the effect of incident shock Mach number has been studied for the $1 \times 1$ and $3 \times 3$ cases. Four different Mach numbers were considered, i.e., $M_{s}=1.1-1.4$ with increments 0.1 . In comparison, a higher center-of-mass velocity is induced for lower incident shock Mach numbers. While contrary to the results of Meng and Colonius (2015), this behavior can be explained by the fact that the induced flow behind the curved reflected shock wave from the top and bottom walls pushes the cylinders of the front column into the opposite direction of the incident shock wave. This upstream pushing force increases as the incident shock Mach number is increased, thus reducing the center-of-mass velocity. Moreover, the transfer of kinetic energy from air to water is faster for weaker shock waves, which is consistent with the velocity observations.

The temperature increment of the flow field has also been investigated. The temperature upstream of the cylinders is higher than downstream, which can be explained by the fact that the gas is expanding isentropically around the 
obstacles. On the other hand, the temperature change in the water is found to be less than $5 \mathrm{~K}$.

The attenuation of the shock wave has been studied by quantitatively measuring the trace of overpressure and impulse at upstream and downstream locations. Upstream of the cylinder matrices, the pressure rises due to the reflected shock. The pressure impulse of the $4 \times 4$ case is the highest, i.e., $I=43.0 \mathrm{~Pa} \cdot \mathrm{s}$. The impulses for the $1 \times 1,2 \times 2$ and $3 \times 3$ cases are $76 \%, 87 \%$ and $94 \%$, respectively, relative to the $4 \times 4$ case. Downstream of the cylinder matrices, the impulses for the $1 \times 1,2 \times 2,3 \times 3$ and $4 \times 4$ cases are $27 \%, 26 \%, 27 \%$ and $23 \%$, compared to the case without cylinders.

Shock attenuation effects of the $1 \times 1$ and $4 \times 4$ cylinders matrices have been compared with the solid obstacle cases with the same geometrical setup. By the end of the simulated time, at the upstream location, the evaluated impulse values of the solid obstacle case are $45 \%$ and $50 \%$ of the water cylinder case relative to the $1 \times 1$ and the $4 \times 4$ cases. At the downstream location, opposite from the trend observed upstream, the impulse values of the water cylinder cases are $40 \%$ and $34 \%$ of the solid obstacle cases with respect to the $1 \times 1$ and the $4 \times 4$ configurations. In all, water cylinders has a better capability than the solid obstacles of the same blockage area to mitigate the shock wave downstream.

Acknowledgements The authors would like to thank the High Performance Computing Center at University of Southern California for providing free access to computing resources.

On behalf of all authors, the corresponding author states that there is no conflict of interest.

\section{References}

Abgrall, R. and Karni, S. (2001). Computations of compressible multifluids. J. Comput. Phys., 169(2):594-623.

Chaudhuri, A., Hadjadj, A., Sadot, O., and Ben-Dor, G. (2013). Numerical study of shock-wave mitigation through matrices of solid obstacles. Shock Waves, 23(1):91-101.

Chauvin, A., Jourdan, G., Daniel, E., Houas, L., and Tosello, R. (2011). Experimental investigation of the propagation of a planar shock wave through a two-phase gas-liquid medium. Phys. Fluids, 23(11):113301.

Chen, H. (2008). Two-dimensional simulation of stripping breakup of a water droplet. AIAA J., 46(5):1135-1143.

Cirak, F., Deiterding, R., and Mauch, S. (2007). Large-scale fluid-structure interaction simulation of viscoplastic and fracturing thin-shells subjected to shocks and detonations. Comput. Struct., 85(11):1049-1065.

Deiterding, R. (2005). An adaptive Cartesian detonation solver for fluidstructure interaction simulation on distributed memory computers. In Parallel Computational Fluid Dynamics-Theory and Application, Proc. Parallel CFD 2005 Conference, pages 333-340. 
Deiterding, R. (2009). A parallel adaptive method for simulating shockinduced combustion with detailed chemical kinetics in complex domains. Comput. Struct., 87(11):769-783.

Deiterding, R. (2011). Block-structured adaptive mesh refinement-theory, implementation and application. ESAIM: Proceedings, 34:97-150.

Deiterding, R., Cirak, F., and Mauch, S. (2009). Efficient fluid-structure interaction simulation of viscoplastic and fracturing thin-shells subjected to underwater shock loading. In Hartmann, S., Meister, A., Schäfer, M., and Turek, S., editors, International Workshop on Fluid-Structure Interaction. Theory, Numerics and Applications.

Flåtten, T., Morin, A., and Munkefjord, S. T. (2011). On solutions to equilibrium problems for systems of stiffened gases. SIAM J. Appl. Math., 71(1):4167.

Gelfand, B. (1996). Droplet breakup phenomena in flows with velocity lag. Prog. Energy Combust. Sci., 22(3):201-265.

Guildenbecher, D., López-Rivera, C., and Sojka, P. (2009). Secondary atomization. Exp. Fluids, 46(3):371.

Igra, D. and Takayama, K. (2001a). Numerical simulation of shock wave interaction with a water column. Shock Waves, 11(3):219-228.

Igra, D. and Takayama, K. (2001b). A study of shock wave loading on a cylindrical water column. Technical report, Report of the Institute of Fluid Science, Tohoku University.

Igra, D. and Takayama, K. (2003). Experimental investigation of two cylindrical water columns subjected to planar shock wave loading. Journal of Fluids Engineering, 125:325-331.

Jourdan, G., Biamino, L., Mariani, C., Blanchot, C., Daniel, E., Massoni, J., Houas, L., Tosello, R., and Praguine, D. (2010). Attenuation of a shock wave passing through a cloud of water droplets. Shock Waves, 20(4):285-296.

Kailasanath, K., Tatem, P., and Mawhinney, J. (2002). Blast mitigation using water - a status report. Technical report, NRL/MR/6400-02-8606, US Naval Research Laboratory.

LeVeque, R. (2002). Finite volume methods for hyperbolic problems. Cambridge University Press, Cambridge.

Meng, J. and Colonius, T. (2015). Numerical simulations of the early stages of high-speed droplet breakup. Shock Waves, 25(4):399-414.

Nomura, K., Koshizuka, S., Oka, Y., and Obata, H. (2001). Numerical analysis of droplet breakup behavior using particle method. J. Nucl. Sci. Technol., 38(12):1057-1064.

Perotti, L., Deiterding, R., Inaba, K., Shepherd, J., and Ortiz, M. (2013). Elastic response of water-filled fiber composite tubes under shock wave loading. Int. J. Solids Struct., 50(3):473-486.

Pilch, M. and Erdman, C. (1987). Use of breakup time data and velocity history data to predict the maximum size of stable fragments for accelerationinduced breakup of a liquid drop. Int. J. Multiph. Flow, 13(6):741-757.

Ranger, A. and Nicholls, J. (1969). Aerodynamic shattering of liquid drops. AIAA J., 7(2):285-290. 
Sembian, S., Liverts, M., Tillmark, N., and Apazidis, N. (2016). Plane shock wave interaction with a cylindrical water column. Phys. Fluids, 28(5):056102.

Shin, Y., Lee, M., Lam, K., and Yeo, K. (1998). Modeling mitigation effects of watershield on shock waves. Shock Vib., 5(4):225-234.

Shyue, K.-M. (1998). An efficient shock-capturing algorithm for compressible multicomponent problems. J. Comput. Phys., 142(1):208-242.

Shyue, K.-M. (2006). A volume-fraction based algorithm for hybrid barotropic and non-barotropic two-fluid flow problems. Shock Waves, 15(6):407-423.

Theofanous, T. (2011). Aerobreakup of newtonian and viscoelastic liquids. Annu. Rev. Fluid. Mech., 43:661-690.

Toro, E., Spruce, M., and Speares, W. (1994). Restoration of the contact surface in the HLL-Rriemann solver. Shock Waves, 4(1):25-34.

Wan, Q. and Eliasson, V. (2015). Numerical study of shock wave attenuation in two-dimensional ducts using solid obstacles: How to utilize shock focusing techniques to attenuate shock waves. Aerospace, 2(2):203-221.

Wan, Q., Jeon, H., Deiterding, R., and Eliasson, V. (2017). Numerical and experimental investigation of oblique shock wave reflection off a water wedge. J. Fluid. Mech., 826:732-758.

Wierzba, A. and Takayama, K. (1988). Experimental investigation of the aerodynamic breakup of liquid drops. AIAA J., 26(11):1329-1335.

Yoshida, T. and Takayama, K. (1990). Interaction of liquid droplets with planar shock waves. J. Fluids Eng., 112(4):481-486. 


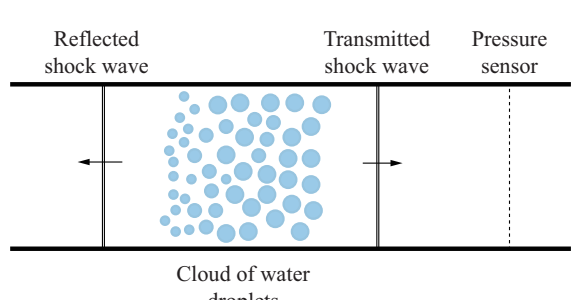

droplets

(a)

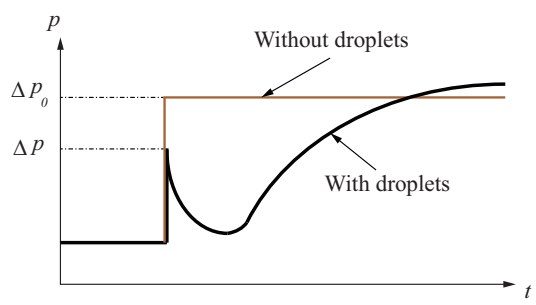

(b)

Fig. 1 Schematic drawing of (a) the propagation of a shock wave through a cloud of water cylinders, and (b) the overpressure trace evaluated at a downstream probe. Figure reproduced from Chauvin et al. (2011). 


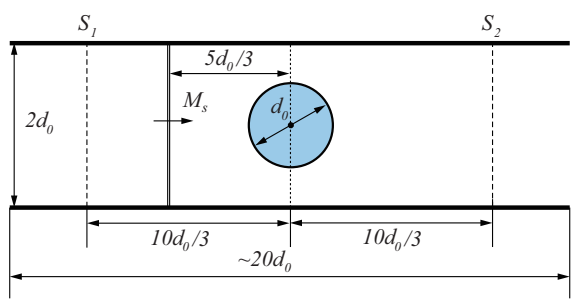

(a) $1 \times 1$ cylinder

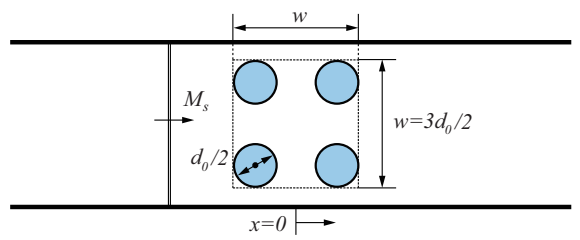

(b) $2 \times 2$ cylinders

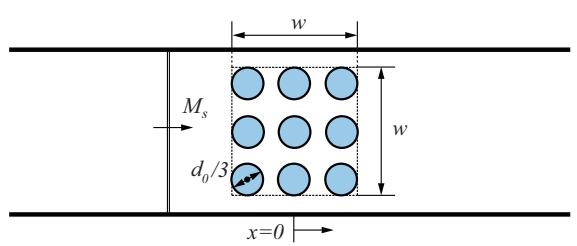

(c) $3 \times 3$ cylinders

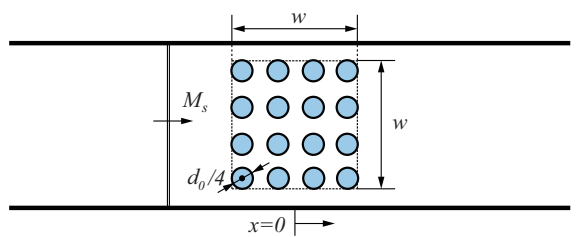

(d) $4 \times 4$ cylinders

Fig. 2 Simulation setup for shock wave propagating through a channel and impact (a) 1 water cylinder, (b) 4 water cylinders, (c) 9 water cylinders, and (d) 16 water cylinders. Not to scale

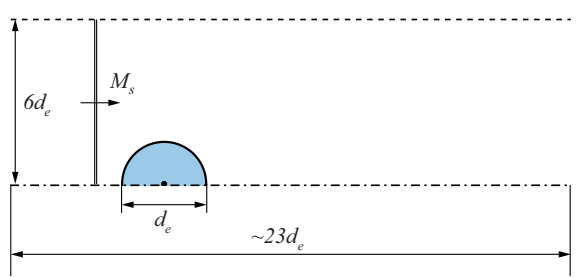

Fig. 3 Simulation setup for numerical comparison to Igra and Takayama (2001b)and Meng and Colonius (2015) 


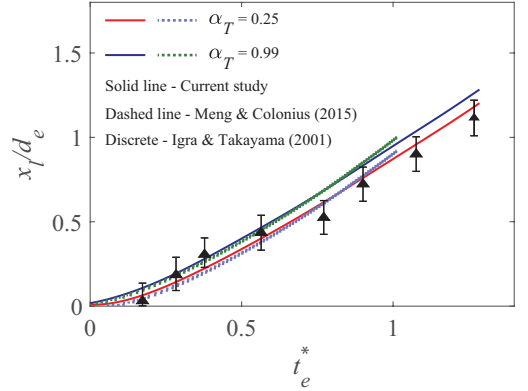

(a) Leading edge drift

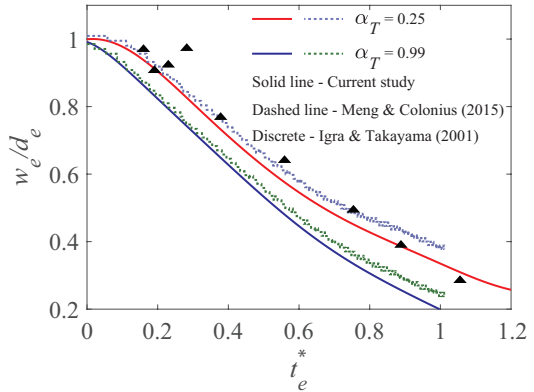

(b) Cylinder centerline width

Fig. 4 (Color online) Evaluation of water cylinder (a) leading edge drift and (b) centerline width. Results from Meng and Colonius (2015) (dashed lines) and Igra and Takayama (2001b) (black triangles) are shown for comparison.

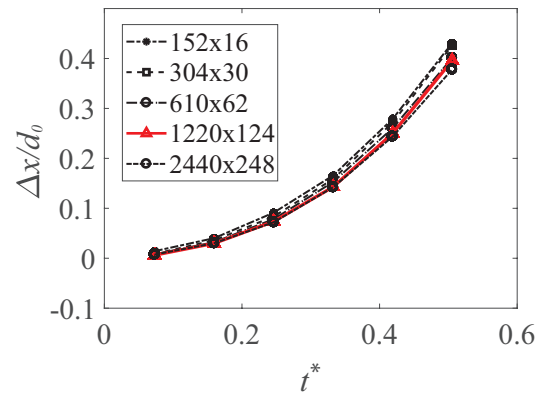

Fig. 5 (Color online) The center-of-mass drift for the 1 water cylinder case. Results obtained with different grid resolutions. 


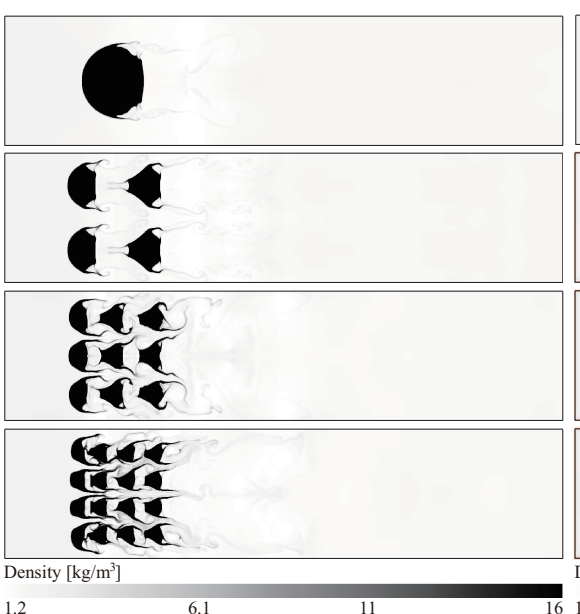

(a) $t=146 \mu \mathrm{s}$

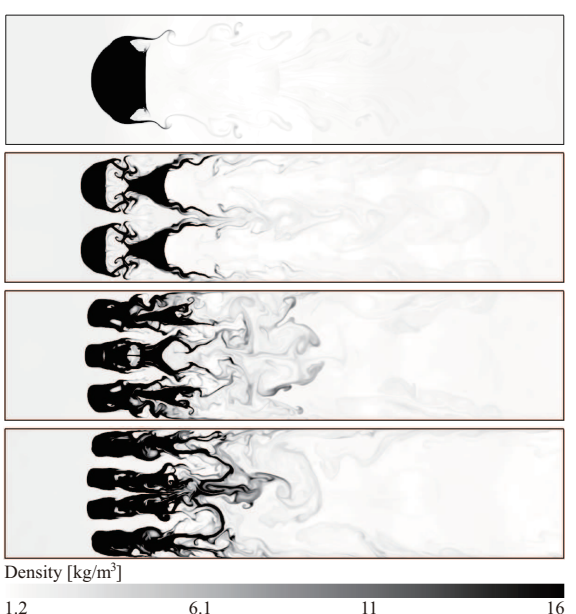

(b) $t=290 \mu \mathrm{s}$

Fig. 6 Density contours of a $M_{s}=1.3$ shock wave impacting $1 \times 1,2 \times 2,3 \times 3$, and $4 \times 4$ water cylinder matrices at (a) $146 \mu \mathrm{s}$, and (b) $290 \mu \mathrm{s}$.

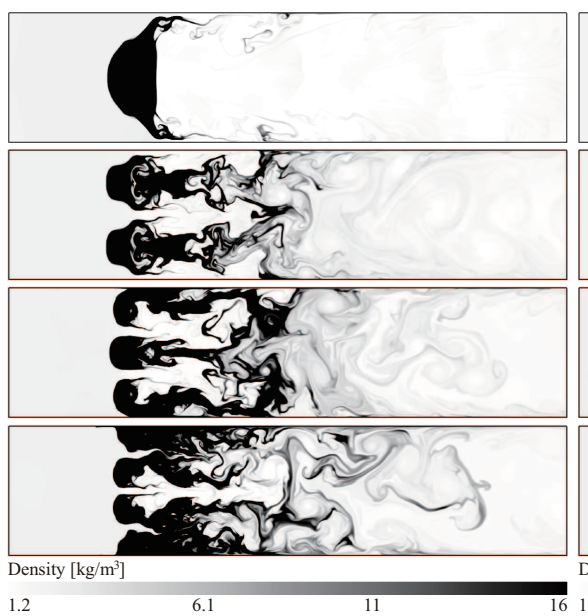

(a) $t=436 \mu \mathrm{s}$

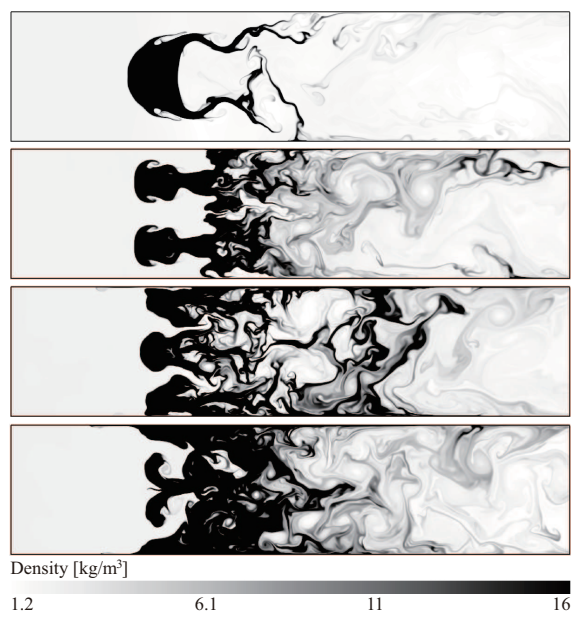

(b) $t=578 \mu \mathrm{s}$

Fig. 7 Density contours of a $M_{s}=1.3$ shock wave impacting $1 \times 1,2 \times 2,3 \times 3$, and $4 \times 4$ water cylinder matrices at (a) $436 \mu \mathrm{s}$, and (b) $578 \mu \mathrm{s}$. 


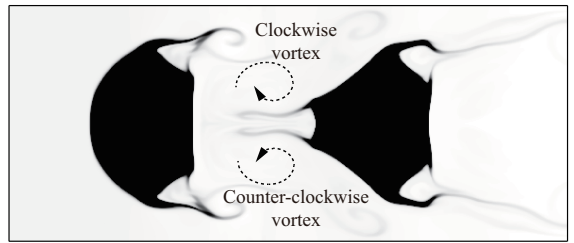

Fig. 8 Water cylinders at the top row for $2 \times 2$ matrix. Density contour at $146 \mu$ s.

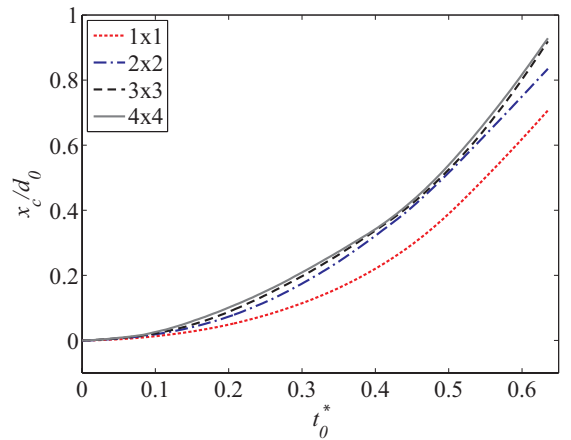

Fig. 9 (Color online) Evaluation of water cylinder matrix center-of-mass drift under incident shock Mach number $M_{s}=1.3$

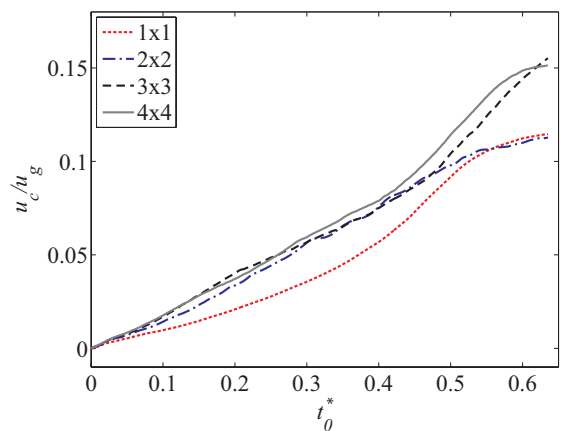

Fig. 10 (Color online) Evaluation of water cylinder matrix center-of-mass velocity under incident shock Mach number $M_{s}=1.3$ 


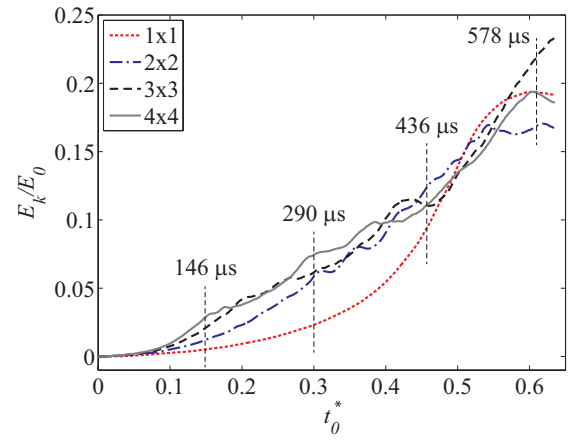

Fig. 11 (Color online) Evaluation of total kinetic energy of water cylinders under the incident shock Mach number $M_{s}=1.3$

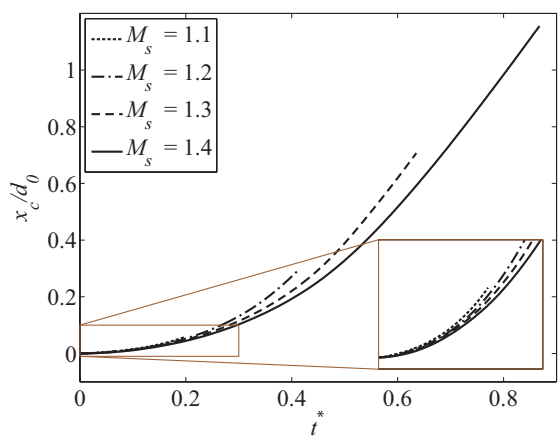

(a) $1 \times 1$ cylinder matrix

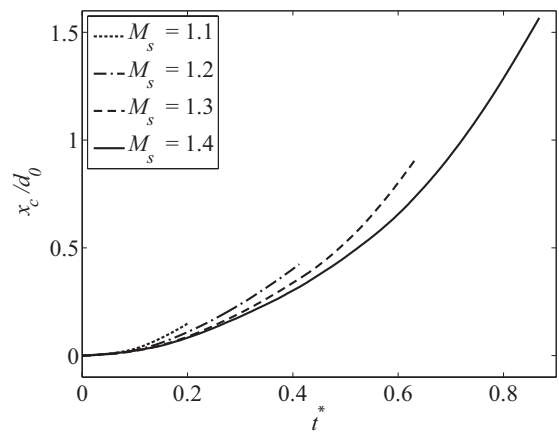

(b) $3 \times 3$ cylinder matrix

Fig. 12 Evaluation of the center-of-mass drift for (a) $1 \times 1$ and (b) $3 \times 3$ water cylinder matrices. Note that the vertical axes have different scaling

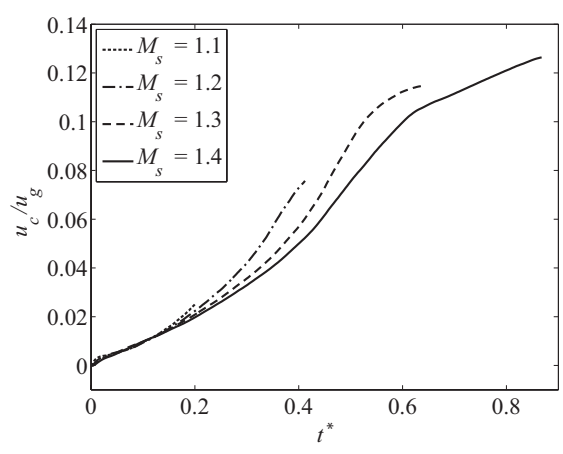

(a) $1 \times 1$ cylinder matrix

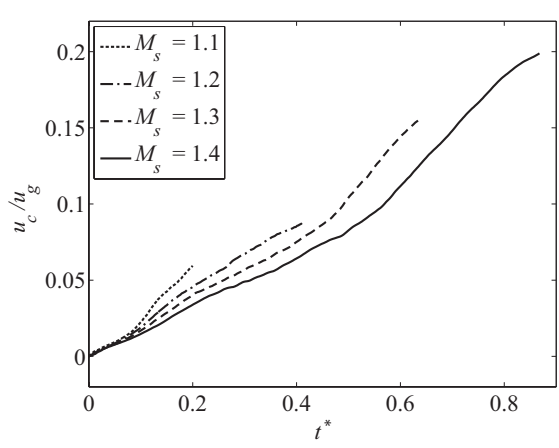

(b) $3 \times 3$ cylinder matrix

Fig. 13 Evaluation of the center-of-mass velocity for (a) $1 \times 1$ and (b) $3 \times 3$ water cylinder matrices. Note that the vertical axes have different scaling 


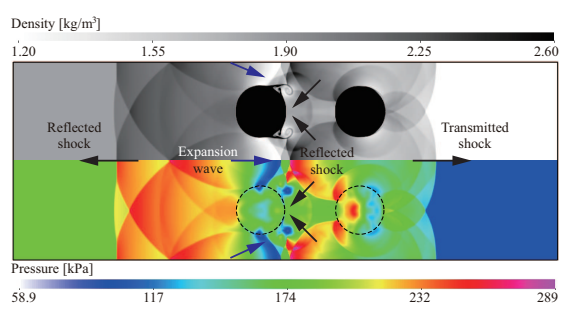

Fig. 14 (Color online) Density (top half) and pressure (bottom half) contours for the propagation of a $M_{s}=1.3$ shock wave through the $2 \times 2$ cylinder configuration at $40 \mu \mathrm{s}$. The shocks reflected from the top and bottom walls move toward the cylinders of the left column.

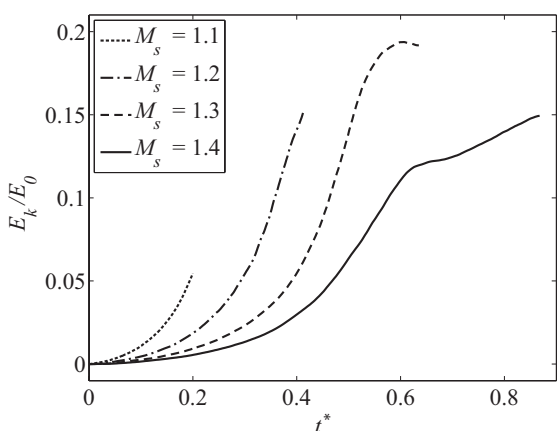

(a) $1 \times 1$ cylinder matrix

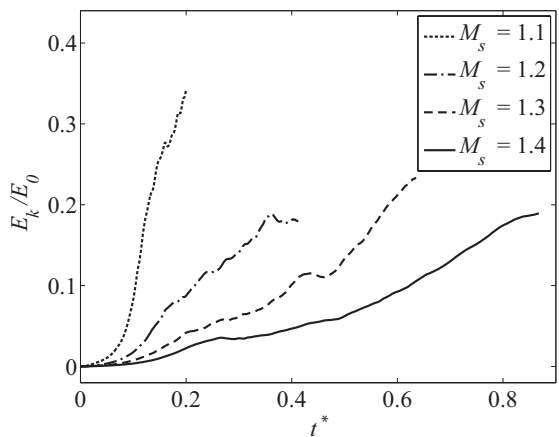

(b) $3 \times 3$ cylinder matrix

Fig. 15 Evaluation of the change of total kinetic energy for (a) $1 \times 1$ and (b) $3 \times 3$ water cylinder matrices

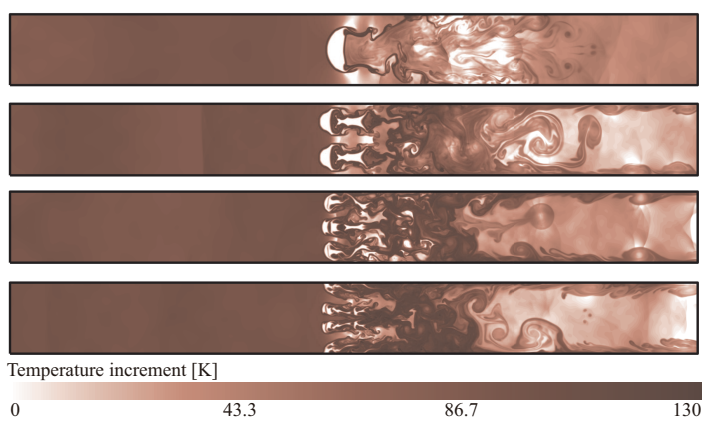

Fig. 16 (Color online) Temperature increment of the flow fields around the water cylinders under the incident shock Mach number $M_{s}=1.3$ at time instant $416 \mu s$ 


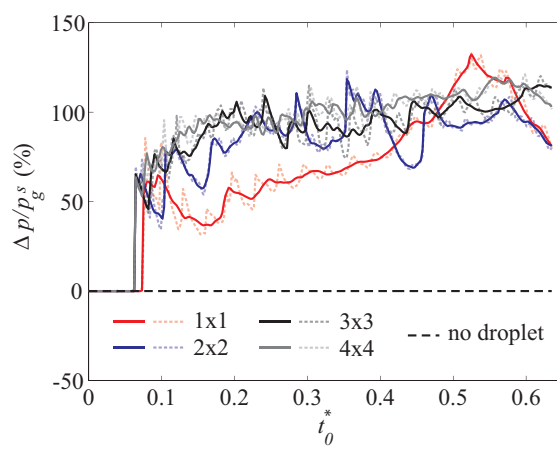

(a) Overpressure

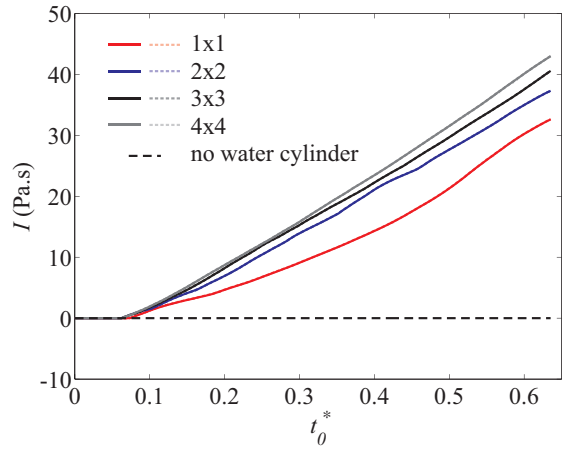

(b) Pressure impulse

Fig. 17 (Color online) Trace of (a) overpressure and (b) pressure impulse (integration of overpressure with regards to time) at upstream location $S_{1}$ under the incident shock Mach number $M_{s}=1.3$. Solid lines denote the trace of averaged evaluation along $S_{1}$, and corresponding dashed lines denote the evaluation at the center point of $S_{1}$. Note that the solid and the corresponding dashed lines in (b) overlap. The theoretical solution of the case without water cylinders is shown for comparison. 


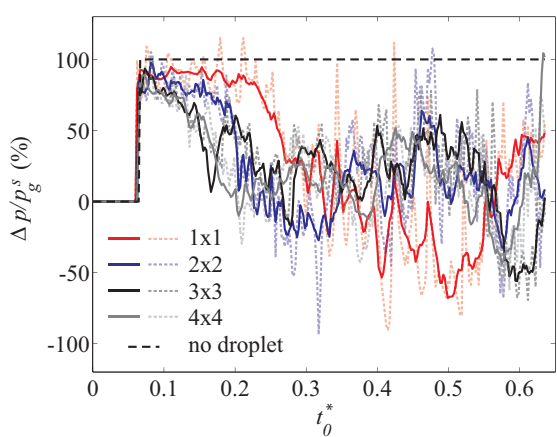

(a) Overpressure

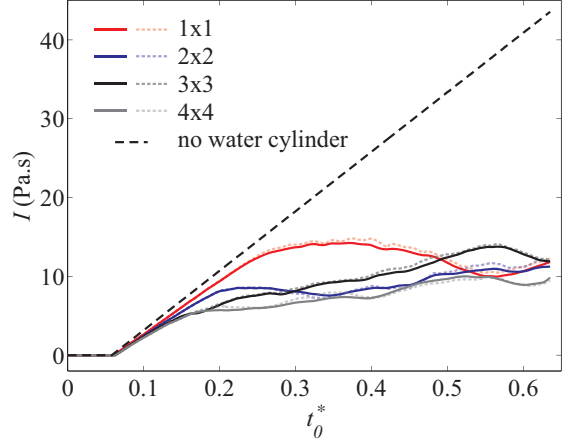

(b) Pressure impulse

Fig. 18 (Color online) Trace of (a) overpressure and (b) pressure impulse at downstream location $S_{2}$ under the incident shock Mach number $M_{s}=1.3$. Solid lines denote the trace of averaged evaluations along $S_{2}$, and corresponding dashed lines denote the evaluations at the center point of $S_{2}$. The theoretical solution of the case without water cylinders is shown for comparison.

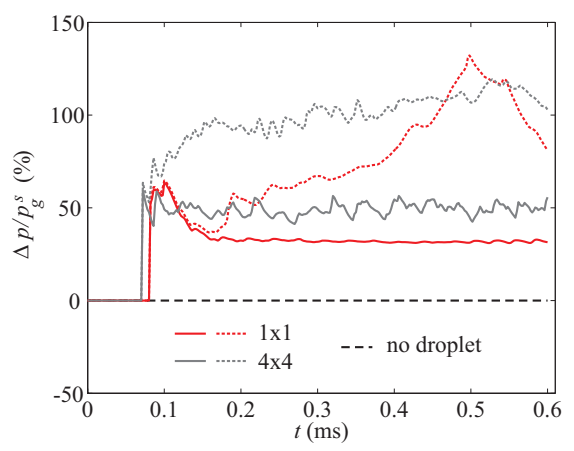

(a) Overpressure

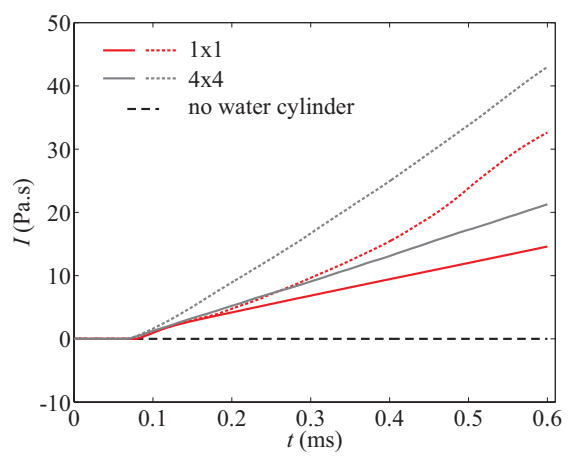

(b) Pressure impulse

Fig. 19 (Color online) Trace of (a) overpressure and (b) pressure impulse (integration of overpressure with regards to time) at upstream location $S_{1}$ under the incident shock Mach number $M_{s}=1.3$. Solid lines denote the traces of the solid obstacle cases, and dashed lines denote the evaluations for water cylinder matrix cases. The theoretical solution of the case without water cylinders is shown for comparison. 


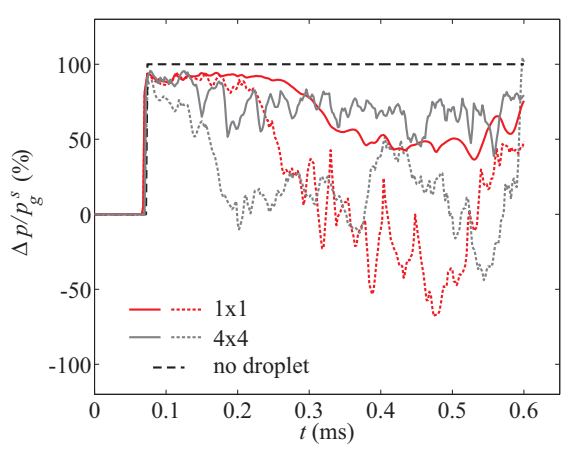

(a) Overpressure

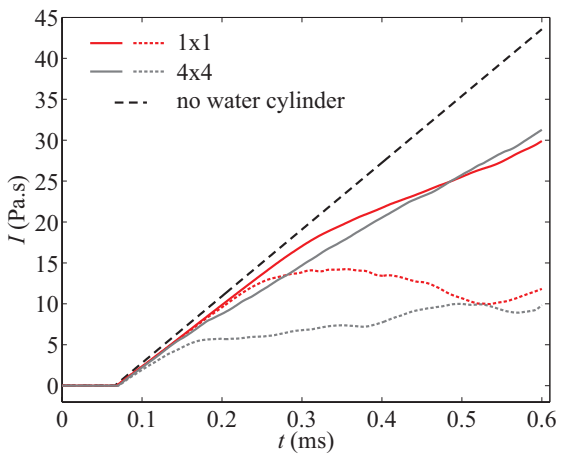

(b) Pressure impulse

Fig. 20 (Color online) Trace of (a) overpressure and (b) pressure impulse at upstream location $S_{2}$ under the incident shock Mach number $M_{s}=1.3$. Solid lines denote the traces of the solid obstacle cases, and dashed lines denote the evaluations for water cylinder matrix cases. The theoretical solution of the case without water cylinders is shown for comparison. 